\title{
Evolución clínica en recién nacidos con presunción de sepsis nosocomial tratados con cefazolina o vancomicina. Estudio de no inferioridad, aleatorizado, controlado Clinical outcome of neonates with nosocomial suspected sepsis treated with cefazolin or vancomycin. A non-inferiority, randomized, controlled trial
}

\author{
Dr. José M. Ceriani Cernadas ${ }^{a, b}$, Dra. Silvia Fernández Jonusas ${ }^{a}$,

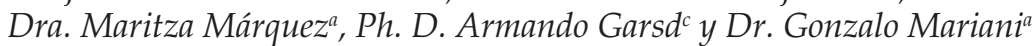 \\ Colaboradores: Dra. Amorina Pardo, Dr. Adrián Aguilar, Dra. Andrea Lew y \\ Dr. Marcelo Armadans
}

\section{RESUMEN}

Las infecciones nosocomiales persisten elevadas en las unidades de cuidados intensivos neonatales, lo cual motiva el muy frecuente uso de antibióticos. Nuestro objetivo fue evaluar la eficacia de la cefazolina frente a la vancomicina como terapia inicial en los recién nacidos con signos clínicos presuntivos de sepsis nosocomial probablemente causada por Staphylococcus coagulasa negativo.

Métodos. Se incluyeron recién nacidos hospitalizados con signos clínicos muy probables de sepsis bacteriana nosocomial. Dos grupos fueron asignados aleatoriamente según el antibiótico utilizado en el inicio del tratamiento: grupo cefazolina (GC) o grupo vancomicina (GV). El análisis primario se realizó mediante la intención de tratamiento. La medida principal de resultado fue la evolución clínica de los neonatos en ambos grupos al final del tratamiento.

Resultados. Se analizaron 109 recién nacidos, 52 en el GC y 57 en el GV. Las características basales fueron similares entre los grupos. El porcentaje de recién nacidos con evolución clínica adecuada fue de $92 \%$ en el GC y de $86 \%$ en el GV: diferencia de $6 \%$ (95\% CI: de $-7 \%$ a $19 \%$, valor $\mathrm{p}$ de no inferioridad, $\mathrm{p}=0,007)$. En el GC, fallecieron 7 recién nacidos $(13,5 \%)$ y 11 , en el GV $(19,2 \%)$, diferencia no significativa $(p=0,45)$. Conclusión. En recién nacidos con sepsis nosocomial confirmada o altamente probable, la cefazolina no fue inferior a la vancomicina en el logro de un resultado clínico adecuado.

Palabras clave: infecciones nosocomiales, neonatos, cefazolina, vancomicina, estafilococo coagulasa negativo.

ClinicalTrials.gov: NCT01867138.

http:/ /dx.doi.org/10.5546/aap.2014.308

Correspondencia: Dr. José M. Ceriani Cernadas: jose.ceriani@ hospitalitaliano.org.ar

Conflicto de intereses: Ninguno que declarar.

Recibido: 28-1-2014 Aceptado: 27-3-2014 a aumentar, principalmente, por la mayor supervivencia de prematuros muy pequeños y la creciente complejidad de los tratamientos invasivos. El Staphylococcus coagulasa negativo (SCN) es el agente causal más frecuente en la sepsis neonatal nosocomial o tardía. Estudios del National Institute of Child Health and Human Development Neonatal Research Network de EE. UU., mostraron que entre el 55 y el 58\% de las bacteriemias nosocomiales en prematuros de muy bajo peso al nacer son producidas por SCN. ${ }^{1,2}$ En otro estudio que recolectó datos de 29 unidades neonatales de cuidado intensivo, el SCN fue responsable de sepsis nosocomial en el $48,3 \%$ de los neonatos ${ }^{3}$ y otro más reciente mostró una tasa de $45 \%{ }^{4}$

La vancomicina es uno de los antibióticos de elección ante la presunción de sepsis por SCN y otros gérmenes Gram positivos, y se emplea en el tratamiento empírico inicial en sepsis tardía o nosocomial, ${ }^{5-7}$ habitualmente asociada a un antibiótico de amplio espectro. Su excesivo uso provoca diversos problemas, posibles efectos adversos, mantenimiento prolongado de vías intravenosas y la aparición de resistencia bacteriana, que es el que ha merecido mayor preocupación en la búsqueda de estrategias para reducirla. ${ }^{8-9}$

Probablemente, una de las principales causas de esta creciente resistencia antimicrobiana es el 
extenso uso de la vancomicina en los hospitales a partir de la emergencia de la resistencia a la meticilina a fines de la década del $70 .{ }^{6}$ Por tal motivo, una de las estrategias recomendadas para reducir su uso es reemplazarla por antibióticos alternativos. ${ }^{8,9}$

A fin de comprobar la utilidad de esta estrategia, realizamos el presente estudio con el principal objetivo de evaluar la eficacia del tratamiento empírico con cefazolina en comparación con vancomicina en neonatos con signos clínicos presuntivos de sepsis bacteriana adquirida en el Hospital, probablemente producida por SCN.

\section{Hipótesis}

Los recién nacidos con diagnóstico presuntivo de sepsis nosocomial, que reciben cefazolina en el tratamiento empírico inicial, presentan una evolución clínica no inferior a la de aquellos tratados con vancomicina.

\section{Diseño del estudio}

Estudio aleatorizado, controlado, de grupos paralelos y de no inferioridad.

\section{POBLACIÓN Y MÉTODOS}

El estudio se realizó en la División de Neonatología del Hospital Italiano de Buenos Aires, Argentina, entre marzo de 2006 y agosto de 2010. Fue patrocinado por la Fundación Carlos A. Gianantonio, entidad sin fines de lucro y no hubo ninguna financiación externa. El Comité de Ética de Protocolos de Investigación del Hospital aprobó el estudio (Protocolo 563) y todos los padres de los niños participantes firmaron el consentimiento informado.

\section{POBLACIÓN}

Resultaron elegibles para el enrolamiento en el estudio los recién nacidos hospitalizados en la UCIN con signos clínicos muy probables de sepsis bacteriana nosocomial o tardía.

Se incluyeron neonatos mayores de 3 días de edad que, a consideración de los médicos a cargo y las guías de la UCIN, tenían indicación de vancomicina en el tratamiento inicial ante la sospecha de sepsis bacteriana y a quienes se les hubiera efectuado al menos un hemocultivo previo al tratamiento antimicrobiano.

Se excluyeron los recién nacidos que, al momento de la inclusión, hubieran sido tratados con vancomicina en la semana previa o fueran derivados de otros centros y a su ingreso estuvieran recibiendo antibióticos.

\section{MÉTODOS}

Asignación de los pacientes al tratamiento: los recién nacidos que cumplían con los criterios de inclusión/exclusión fueron asignados en forma aleatoria a dos grupos de acuerdo con el siguiente esquema antibiótico de inicio: cefazolina y amikacina [grupo cefazolina (GC)] o vancomicina y amikacina [grupo vancomicina (GV)].

Los antibióticos se administraron por vía intravenosa en dosis e intervalos acordes a la edad gestacional y posnatal. Los recién nacidos recibieron los controles y estudios auxiliares que se realizan ante la presunción de sepsis bacteriana.

La duración de la terapia fue de 7 a 10 días de acuerdo con la evolución clínica y con la presencia de un segundo hemocultivo positivo a las $72 \mathrm{~h}$ de haber comenzado el tratamiento. Si el curso clínico fue satisfactorio con normalización de las pruebas de laboratorio inicialmente alteradas, los antibióticos fueron suspendidos a los siete días de tratamiento. En los recién nacidos que tuvieron un segundo hemocultivo positivo o una evolución más tórpida de acuerdo con la consideración del médico a cargo de la UCIN, el tratamiento continuó hasta los 10 días.

Variable principal de resultado: evolución clínica de los recién nacidos de ambos grupos en el episodio de sepsis nosocomial confirmada o muy probable que motivó su incorporación al estudio. (Véanse detalles bajo el subtítulo "Evaluación clínica").

Variable secundaria de resultado: porcentaje de recién nacidos asignados al grupo cefazolina que pasaron a recibir vancomicina en reemplazo de cefazolina.

\section{Definiciones}

Se definió sepsis confirmada cuando, ante un cuadro clínico compatible con sepsis, el hemocultivo y/o líquido cefalorraquídeo (LCR) fueron positivos.

Se describió sepsis muy probable cuando los cultivos eran negativos y el recién nacido presentaba signos clínicos de sepsis y dos o más de los siguientes resultados en pruebas diagnósticas: < 5000 glóbulos blancos $/ \mathrm{mm}^{3}$, $<1500$ neutrófilos $/ \mathrm{mm}^{3}$, relación neutrófilos inmaduros sobre neutrófilos totales (NI/NT) $\geq 0,2$, proteína $C$ reactiva $>10 \mathrm{mg} / \mathrm{L}$ y número de plaquetas inferior a $100000 / \mathrm{mm}^{3}$.

Se consideró sepsis nosocomial o tardía cuando los signos clínicos de infección bacteriana se presentaron luego del tercer día de vida y hasta antes del alta. 


\section{Evaluación clínica}

La evaluación clínica para responder a la variable principal de resultados se efectuó entre los 7 y los 10 días de haber comenzado el tratamiento. Cada neonato fue evaluado por dos de los investigadores en forma independiente para definir si la evolución clínica era adecuada o inadecuada. A fin de lograr una mayor objetividad, la evaluación clínica se efectuó mediante los siguientes criterios:

Adecuada, cuando el neonato presentaba normalidad de todos los parámetros clínicos, buen estado general, apropiada tolerancia al alimento por vía gástrica, control normal de la temperatura, cultivos negativos en sangre y LCR, y normalización de los resultados de los análisis de laboratorio efectuados al comienzo del cuadro clínico.

Inadecuada, cuando estaban presentes uno o más de los siguientes aspectos: evolución clínica con persistencia de algún signo de sepsis, hemocultivo o LCR positivo después de las $72 \mathrm{~h}$ de haber comenzado el tratamiento, persistencia de pruebas de laboratorio anormales y muerte por sepsis.

\section{Aleatorización \\ El proceso de aleatorización fue realizado y supervisado por uno de los investigadores (JMCC), que no estuvo involucrado en el cuidado clínico de los pacientes en estudio. El listado fue generado a través de un programa informático, mediante la creación de bloques aleatorios de tamaño variable $(6,8$ y 10$)$.Los detalles de la asignación del grupo de tratamiento fueron guardados en sobres opacos con una numeración secuencial. La asignación de los grupos se mantuvo oculta hasta que se obtuvo el consentimiento escrito de los padres.}

\section{Cambio en la asignación del tratamiento antibiótico}

En los pacientes con inadecuada respuesta a la cefazolina, en las $72 \mathrm{~h}$ de haber comenzado el tratamiento, se realizó un cambio y fueron asignados a recibir vancomicina. Esta conducta fue delineada en un algoritmo bien definido. A efectos de minimizar sesgos, el neonatólogo a cargo del paciente debía consultar con el jefe de la UCIN y con uno de los investigadores del estudio para contar con su aprobación antes de tomar la decisión final de modificar el tratamiento.

\section{Tamaño de la muestra}

Para los fines del cálculo del tamaño de la muestra, se asumió primariamente una tasa de adecuada respuesta al tratamiento en ambos grupos de $90 \%$. Con el uso de una aproximación normal de distribución binomial, se requerirían 112 pacientes en cada brazo de tratamiento para demostrar que el margen superior del intervalo de confianza del $95 \%$ para la diferencia de los tratamientos en la tasa de evolución clínica adecuada fuera menor de $10 \%$ (poder $80 \%$; $\alpha=0,05) \cdot{ }^{10,11}$ Teniendo en cuenta la magnitud del tamaño de la muestra, se decidió efectuar un abordaje adaptativo del estudio en dos pasos secuenciales con ajustes estadísticos para los múltiples análisis. ${ }^{12}$ En una primera etapa, se debían aleatorizar 115 neonatos y, si los resultados confirmaban la hipótesis, el estudio finalizaba en esta etapa.

Sin embargo, si no era así pero los resultados tentativos eran promisorios y resultaba factible reclutar el total de pacientes calculados, el estudio continuaba en una segunda etapa luego de una nueva estimación del tamaño muestral. ${ }^{13,14}$

\section{Análisis estadístico}

La exposición principal fue el tratamiento antibiótico en neonatos con sepsis nosocomial. El análisis primario fue realizado por intención de tratamiento. Varios análisis de sensibilidad también fueron efectuados. La comparación de la eficacia entre los grupos de tratamiento se realizó considerando las diferencias en la tasa de respuesta en ambos grupos y el IC del 95\% de esta diferencia en relación con la tolerancia a la no inferioridad y basado en el enfoque de margen fijo. ${ }^{15}$ Las medidas de resultado binomiales fueron probadas mediante la prueba de Wald con corrección de continuidad.

Para todos los análisis, se empleó el SAS software versión 9.3 (SAS Institute Inc., Cary, CN, EE. UU.).

\section{RESULTADOS}

El total de pacientes analizados fue 109 , de los cuales 52 pertenecían al grupo cefazolina y 57 al grupo vancomicina (Figura 1). Los grupos fueron homogéneos en sus características basales (Tabla 1).

Ambos grupos presentaron signos clínicos de sepsis similares (Tabla 2).

No hubo diferencias en el porcentaje de sepsis confirmada entre los grupos: $38,4 \%(20 / 52)$ en el grupo cefazolina y $38,6 \%(22 / 57)$ en el grupo vancomicina (Tabla 3).

Asimismo, también fue similar el porcentaje de sepsis confirmada producida por SCN: 70,0\% $(14 / 20)$ en el GC y $72,7 \%(16 / 22)$ en el GV. 


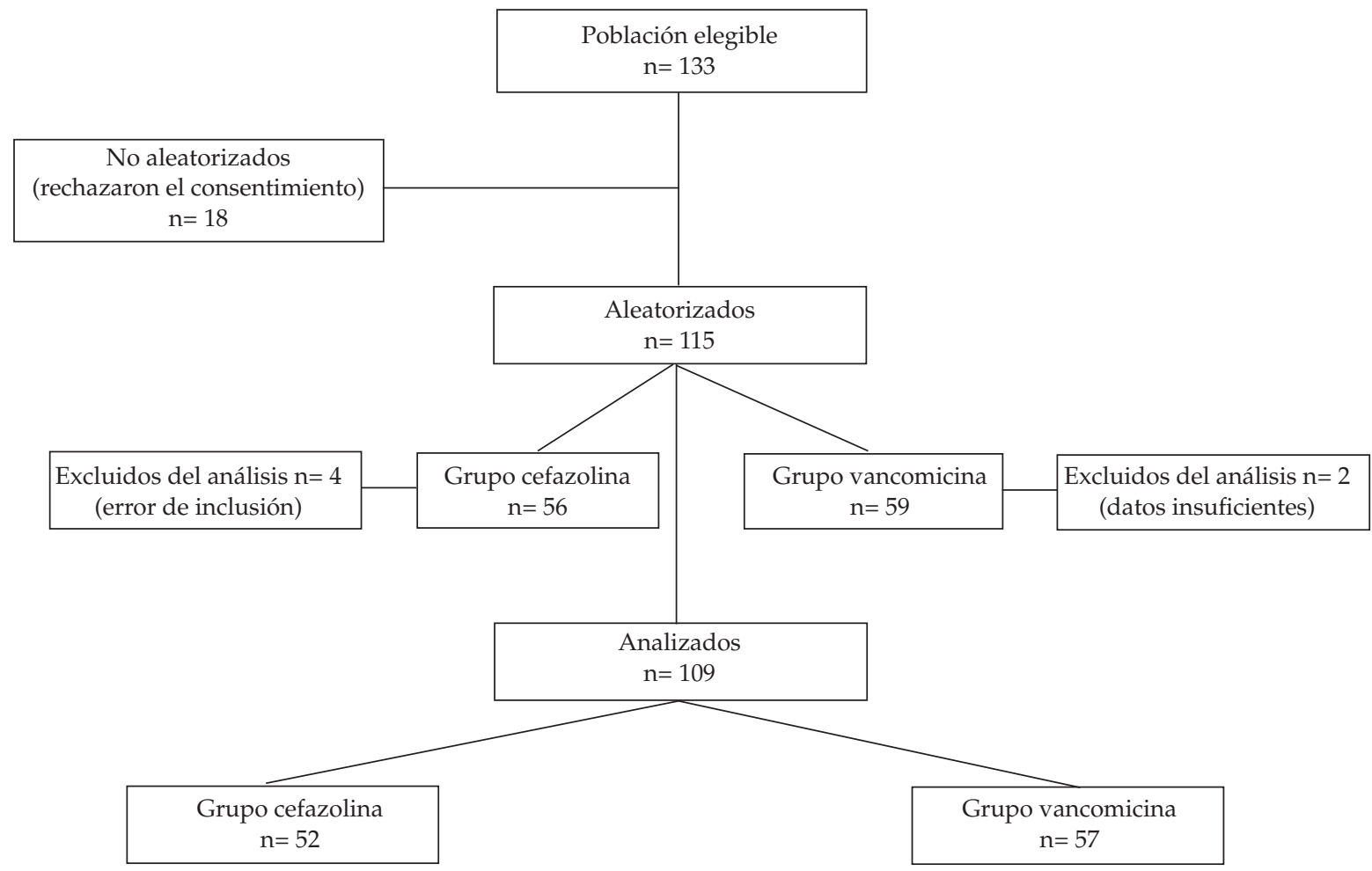

TABla 1. Características de la población

\begin{tabular}{lccc}
\hline & Grupo cefazolina N= 52 & Grupo vancomicina N= 57 & $\mathbf{p}^{*}$ \\
\hline Morbilidad materna en el embarazo, n (\%) & $21(40,4)$ & $23(40,3)$ & 1,0 \\
Embarazo gemelar, n (\%) & $4(7,7)$ & $4(7,0)$ & 1,0 \\
Recién nacidos derivados, n (\%) & $9(17,3)$ & $12(21,0)$ & 0,64 \\
Edad gestacional, semanas, media (DE) & $31,2(5,1)$ & $31,5(4,7)$ & 0,83 \\
Prematurez (<37 semanas), n (\%) & $42(80,7)$ & $47(82,4)$ & 1,0 \\
RCIU, n (\%) & $7(13,4)$ & $4(7,0)$ & 0,35 \\
Peso al nacer, g, media (DE) & $1629,3(1001,5)$ & $1650,1(888,9)$ & 0,91 \\
Apgar 5 minutos $<7, \mathrm{n}(\%)$ & $3(5,7)$ & $5(8,7)$ & 0,72 \\
\hline
\end{tabular}

RCIU: restricción del crecimiento intrauterino.

* Significado estadístico de las respectivas diferencias, basado en el test de chi cuadrado exacto para la diferencia en las proporciones binomiales y la prueba de Student de dos muestras para la diferencia en la edad gestacional y el peso al nacer, respectivamente.

TABLA 2. Signos clínicos más frecuentes de sepsis en ambos grupos

\begin{tabular}{lccc}
\hline Signo clínico (N, \%) & Grupo cefazolina $\mathbf{N = 5 2}$ & Grupo vancomicina $\mathbf{N}=\mathbf{5 7}$ & $\mathbf{P}^{*}$ \\
\hline Distensión abdominal y/o residuo & $21(40,3)$ & $18(31,8)$ & 0,42 \\
Hipertermia & $19(36,5)$ & $23(40,3)$ & 0,70 \\
Apneas & $17(32,6)$ & $18(31,5)$ & 1,0 \\
Dificultad respiratoria & $17(32,6)$ & $24(42,1)$ & 0,33 \\
Taquicardia & $14(26,9)$ & $11(19,3)$ & 0,37 \\
\hline
\end{tabular}

*Significado estadístico de las respectivas diferencias, basado en el test de chi cuadrado exacto para la diferencia en las proporciones binomiales. 
Tres recién nacidos del GC $(5,7 \%)$ y cinco del GV $(9,4 \%)$ presentaron, a las $72 \mathrm{~h}$, un segundo hemocultivo positivo a SCN.

Otras bacterias halladas en neonatos de ambos grupos están señaladas en la Tabla 3.

No se observaron efectos adversos asociados con el uso de los antibióticos, excepto en un recién nacido del grupo vancomicina, que, después de 7 días de tratamiento, presentó un hemocultivo positivo a Candida albicans y debió ser medicado con anfotericina B.

La mortalidad global fue de $13,5 \%(7 / 52)$ en el GC y $19,2 \%(11 / 57)$ en el GV $(p=0,45)$ y la tasa de mortalidad por sepsis fue de $4 \%$ y $9 \%$ en los grupos cefazolina y vancomicina, respectivamente $(\mathrm{p}=0,44)$.

\section{Variable principal de resultado}

En el análisis por intención de tratamiento, la evolución clínica fue adecuada en el 92,3\% (48/52) de los pacientes del GC y en el 86,0\% (49/57) del GV. La diferencia en el porcentaje, 6,3\% (95\% IC: de $-7,1 \%$ a $19,8 \%$ ), es estadísticamente significativa en la prueba de no inferioridad: $p=0,007$. El análisis por protocolo confirmó la significancia de estos datos $(\mathrm{p}<0,001)$.

La proporción de evolución clínica adecuada en sepsis por SCN también mostró valores similares en ambos grupos: 92,9\% (13/14) en el GC y $87,5 \%$ (14/16) en el GV, aunque el bajo tamaño muestral no permitió asegurar la paridad entre los dos antibióticos (95\% IC: de $-22,4 \%$ a $33,1 \%$; valor de no inferioridad: $\mathrm{p}=0,21$ ).

\section{Variable secundaria de resultado}

El porcentaje de neonatos asignados al grupo cefazolina que debieron recibir vancomicina fue de $17,3 \%$ (9/52 [IC 95\%: de $8,23 \%$ a $30,33 \%$ ]). En dos de ellos, el cambio se debió a la inadecuada evolución clínica a las 72 h y, en dos, a haber presentado el segundo hemocultivo positivo a las $72 \mathrm{~h}$. Los otros cinco cambios se debieron a la presencia de infección por otros gérmenes.

\section{DISCUSIÓN}

Los resultados de este estudio apoyan la hipótesis de no inferioridad del uso de cefazolina comparada con vancomicina en el tratamiento empírico inicial en recién nacidos con presunción de sepsis nosocomial o tardía. La evolución clínica adecuada entre los 7 y los 10 días de haber comenzado el tratamiento fue similar en ambos grupos. Sin embargo, debe notarse que, aunque la diferencia no fue significativa, la mortalidad por sepsis fue el doble en el grupo que recibió vancomicina.

La vancomicina es uno de los antibióticos de más frecuente elección empírica ante la sospecha de sepsis neonatal tardía, ${ }^{6,7}$ aunque no hay evidencias sólidas en estudios aleatorizados en favor de que un particular antibiótico sea más adecuado que otro en el tratamiento, y aún se desconocen varios aspectos de su farmacología y seguridad en neonatos. ${ }^{16}$ No obstante, su empleo se ha incrementado notablemente en los últimos 20 años. ${ }^{6,9,16}$

Entre los mayores problemas del excesivo uso de la vancomicina, se destaca la resistencia bacteriana de gérmenes Gram positivos, y su incremento es un aspecto de gran preocupación. 89,16

La primera publicación de resistencia bacteriana adquirida a los antibióticos glicopéptidos fue reportada en SCN a mediados

Tabla 3. Diagnóstico de sepsis

\begin{tabular}{lccc}
\hline $\begin{array}{l}\text { Grado de certeza en el } \\
\text { diagnóstico de sepsis } \mathbf{y} \\
\text { patógenos identificados }(\mathbf{n}, \mathbf{\%})\end{array}$ & $\begin{array}{c}\text { Grupo cefazolina } \\
\mathbf{N}=\mathbf{5 2}\end{array}$ & $\begin{array}{c}\text { Grupo vancomicina } \\
\mathbf{N}=\mathbf{5 7}\end{array}$ & $\mathbf{p}$ \\
\hline Confirmada & $20(38,5)$ & $22(38,6)$ & 1,0 \\
Hemocultivo + & $19 / 52(36,5)$ & $22 / 57(38,6)$ & 0,85 \\
LCR & $2 / 45(4,4)^{*}$ & $1 / 48(2,1)^{* *}$ & 0,61 \\
SCN & $14(26,9)$ & $16(28,0)$ & 1,0 \\
Staphylococcus aureus & $3(5,8)$ & $2(3,5)$ & 0,66 \\
Bacilos Gram negativos & $3(5,8)$ & $4(7)$ & 0,64 \\
\hline Muy probable & $32(61,5)$ & $35(61,4)$ & 0,98 \\
\hline
\end{tabular}

LCR: líquido cefalorraquídeo.

SCN: Staphylococcus coagulasa negativo.

* En 45 neonatos, se analizó el LCR; en dos, fue + y, en un recién nacido, el hemocultivo y el LCR fueron +.

** En 48 neonatos, se analizó CSF; en uno, el hemocultivo y el LCR fueron +. 
de la década del $80 \cdot{ }^{17}$ La aparición de la resistencia a la vancomicina en los enterococos se publicó en $1988^{18}$ y rápidamente el interés se concentró en esa resistencia debido a su mayor frecuencia que la resistencia del SCN. Eso llevó a que los mecanismos bioquímicos y genéticos de la resistencia en enterococos fueran rápida y exhaustivamente dilucidados, a diferencia de los mecanismos de resistencia en el SCN, que aún permanecen con varias incertidumbres. En los años siguientes a la primera publicación, los enterococos resistentes a la vancomicina se convirtieron en los principales patógenos nosocomiales, especialmente en los EE. UU. ${ }^{19}$ y la mayoría de las publicaciones estuvo centrada en esa resistencia, incluso en neonatos, ${ }^{20} \mathrm{y}$, asimismo, en la del Staphylococcus aureus, que también emergía en esos años. ${ }^{21}$

En años posteriores, se continuaron describiendo casos de $\mathrm{SCN}$ resistentes ${ }^{16} \mathrm{y}$ también se aislaron cepas con escasa susceptibilidad y parcialmente sensibles a la vancomicina, ${ }^{22-24}$ lo cual tal vez explicaría la permanencia de una inadecuada evolución clínica y bacteriemias prolongadas en algunos neonatos con sepsis producida por SCN. Una menor susceptibilidad a la vancomicina también se describió en infecciones producidas por Staphylococcus aureus. ${ }^{25}$

Se ha observado que la resistencia del SCN a la vancomicina predomina en las cepas Staphylococcus haemolyticus y Staphylococcus epidermidis. ${ }^{24}$ Esta última es una de las cepas más frecuentes en la sepsis neonatal tardía. Además, informes de algunos hospitales han señalado que la susceptibilidad reducida a la vancomicina sería más frecuente entre los SCN resistentes a la meticilina. ${ }^{24}$

El riesgo de un incremento de la resistencia bacteriana a los antibióticos, que constituye un grave problema de salud pública, motivó la implementación de diversas estrategias, en general, basadas en adoptar una conducta en el uso racional de antibióticos, restringiendo sus indicaciones y empleándolos por el menor tiempo posible. ${ }^{8}$ La aplicación de esta medida tiene ciertas dificultades en las unidades neonatales, debido, en especial, a la escasa especificidad del diagnóstico clínico de sepsis, lo cual motiva con alta frecuencia la indicación de antibióticos por "sospecha de sepsis".

Otra estrategia recomendada es el reemplazo de vancomicina por antibióticos alternativos que tengan menor probabilidad de resistencia. 5,6,9,26,27
Nosotros optamos en este estudio por esta última estrategia y evaluamos el reemplazo de vancomicina por un antibiótico de las cefalosporinas de primera generación, basados en estudios previos que mostraron su eficacia en sepsis neonatal producida por SCN y Staphylococcus aureus. 5,6,24

Krediet et al. ${ }^{5}$ observaron en un grupo de recién nacidos con sepsis producida por $\mathrm{SCN}$, 90\% portadores del gen mec A que determina la resistencia a la meticilina, que la cefalotina mostró igual eficacia que la vancomicina en la evolución clínica de los neonatos tratados. Con la estrategia de usar cefalotina, pudieron reducir el uso de vancomicina de $62 \%$ a $21 \%$ en el lapso de 2 años.

Asimismo, Brandt ${ }^{28}$ et al. observaron, en endocarditis experimental por SCN, que la asociación de cefazolina o cefpirona, con rifampicina, mostró igual eficacia que la asociación con vancomicina.

\section{CONCLUSIÓN}

Aun cuando el tamaño de la muestra del presente estudio puede ser considerado una limitación, igualmente nos permitió apoyar la hipótesis de que la cefazolina no es inferior a la vancomicina para lograr una adecuada evolución clínica en recién nacidos con sepsis nosocomial confirmada o muy probable. Por lo tanto, la cefazolina podría reemplazar a la vancomicina en el tratamiento empírico inicial.

No obstante, debido a que se trató de un estudio exploratorio, estos resultados deben ser tomados con precaución hasta que otras investigaciones confirmen nuestros hallazgos.

\section{Agradecimientos}

Los autores agradecemos a los Dres. Ian Scott y Charles Dowding por la revisión crítica del manuscrito. También deseamos agradecer especialmente a los padres de los recién nacidos enrolados en el estudio por haber confiado en nosotros.

\section{BIBLIOGRAFÍA}

1. Stoll BJ, Hansen N, Fanaroff AA, Wright LL, et al. Late-onset sepsis in very low birth weight neonates: the experience of the NICHD Neonatal Research Network. Pediatrics 2002;110(2):285-91.

2. Fanaroff AA, Korones SB, Wright LL, Verter J, et al. Incidence, presenting features, risk factors and significance of late onset septicemia in very low birth weight infants. Pediatr Infect Dis J 1998;17(7):593-8.

3. Sohn AH, Garrett DO, Sinkowitz-Cochran RL, Grohskopf LA, et al. Prevalence of nosocomial infections in neonatal 
intensive care units patients: results from the first national point-prevalence survey. J Pediatr 2001;139(6):821-7.

4. Muller-Pebody B, Johnson AP, Heath PT, Gilbert RE, et al. Empirical treatment of neonatal sepsis: are the current guidelines adequate? Arch Dis Child Fetal Neonatal Ed 2011;96(1):F4-8.

5. Krediet TG, Jones ME, Gerards LJ, Fleer A. Clinical outcome of cephalothin versus vancomycin therapy in the treatment of coagulase-negative staphylococcal septicemia in neonates: relation to methicillin resistance and mec A gene carriage of blood isolates. Pediatrics 1999;103(3):E29.

6. Venkatesh MP, Placencia F, Weisman LE. Coagulasenegative staphylococcal infections in the neonate and child: an update. Semin Pediatr Infect Dis 2006;17(3):120-7.

7. Gordon A, Jeffery HE. Antibiotic regimens for suspected late onset sepsis in newborn infants. Cochrane Database Syst Rev 5005;(3):CD004501.

8. Issacs D. Rationing antibiotic use in neonatal units. Arch Dis Child Fetal Neonatal Ed 2000;82(1):F1-2.

9. Chiu $\mathrm{CH}$, Michelow IC, Cronin J, Ringer SA, et al. Effectiveness of a guideline to reduce vancomycin use in the neonatal intensive care unit. Pediatr Infect Dis J 2011;30(4):273-8.

10. Blackwelder WC. "Proving the null hypothesis" in clinical trials. Control Clin Trials 1982;3(4):345-53.

11. Sachs L. Applied statistics: a handbook of techniques. 2nd ed. New York: Springer-Verlag;1984. Pág.333.

12. Blackwelder WC, Chang MA. Sample size graphs for "proving the null hypothesis". Control Clin Trials 1984;5(2):97-105.

13. Denne JS. Estimation following extension of a study on the basis of conditional power. J Biopharm Stat 2000;10(2):13144.

14. Mehta CR, Pocock SJ. Adaptive increase in sample size when interim results are promising: A practical guide with examples. Stat Med 2011;30(28):3267-84.

15. LiG, ChiGYH. Inferiority indexand margininnoninferiority trials. Stat Biopharm Res 2011;3(2):288-301.

16. Dehority W. Use of vancomycin in pediatrics. Pediatr Infect Dis J 2010;29(5):462-4.

17. Schwalbe RS, Stapleton JT, Gilligan PH. Emergence of vancomycin resistance in coagulase-negative staphylococci. N Engl J Med 1987;316(15):927-31.
18. Uttley AH, Collins CH, Naidoo J, Giorge RC. Vancomycinresistant enterococci. Lancet 1988;1:57-8.

19. Centers for Disease Control and Prevention. Nosocomial enterococci resistant to vancomycin-United States, 19891993. MMWR 1997;42(30):597-9.

20. Sherer CR, Sprague BM, Campos JM, Nambiar S, et al. Characterizing vancomycin-resistant enterococci in neonatal intensive care. Emerg Infect Dis 2005;11(9):1470-2.

21. Smith TL, Pearson ML, Wilcox KR, CruzC, etal. Emergence of vancomycin resistance in Staphylococcus aureus. Glycopeptide-Intermediate Staphylococcus aureus Working Group. N Engl J Med 1999;340(7):493-501.

22. Sieradzki K, Roberts RB, Haber SW, Tomasz A. The development of vancomycin resistance in a patient with methicillin-resistant Staphylococcus aureus infection. NEngl J Med 1999;340(7):517-23.

23. Center KJ, Reboli AC, Hubler R, Rotgers GL, Long SSl. Decrease vancomycin susceptibility of coagulase-negative staphylococci in a neonatal intensive care unit: evidence of spread of Staphylococcus warneri. J Clin Microbiol 2003;41(10):4660-5.

24. BiavascoF, VignaroliC,VaraldoPE. Glycopeptideresistance in coagulase-negative staphylococci. Eur J Microbiol Infect Dis 2000;19(6):403-17.

25. van HalSJ, Lodice TP, Paterson DL. The clinical significance of vancomycin minimum inhibitory concentration in Staphylococcus aureus infections: a systematic review and meta-analysis. Clin Infect Dis 2012;54(6):755-71.

26. Karlowicz MG, Buescher ES, Surka AE. Fulminant lateonset sepsis in a neonatal intensive care unit, 1988-1997, and the impact of avoiding empiric vancomycin therapy. Pediatrics 2000;106(6):1387-90.

27. Lawrence SL, Roth V, Slinger R, Toye B, et al. Cloxacillin versus vancomycin for presumed late-onset sepsis in the Neonatal Intensive Care Unit and the impact upon outcome of coagulase negative staphylococcal bacteremia: a retrospective cohort study. BMC Pediatr 2005;5:49.

28. Brandt CM, Rouse MS, Tallan BM, Laue NW, et al. Effective treatment of cephalosporin-rifampin combinations against cryptic methicillin-resistant beta-lactamaseproducing coagulase-negative staphylococcal experimental endocarditis. Antimicrob Agents Chemother 1995;39(8):1815-9. 